\title{
Perfil do EMG em relação a duas angulações distintas durante a contracção voluntária isométrica máxima no exercício de agachamento
}

\author{
EMG profile in two different angles during maximum voluntary isometric \\ contraction in squat exercise
}

\author{
A.S. Maior, L. Marmelo, S. Marques-Neto
}

\begin{abstract}
O objetivo do estudo foi verificar a atividade elétrica dos músculos vasto medial (VM) e do vasto lateral (VL) em duas angulações distintas $\left(70^{\circ}\right.$ e $90^{\circ}$ de flexão de joelho) durante a contração voluntária isométrica máxima (CVIM) realizada no exercício de agachamento. Foram selecionados 15 sujeitos do sexo masculino, voluntários e destreinados há mais de 12 meses em relação ao treinamento de força (idade: $28.0 \pm 4.6$ anos, estatura: $180.0 \pm 5.2 \mathrm{~cm}$, massa corporal: $80.0 \pm 3.2 \mathrm{~kg}$ e IMC: $24.2 \pm 1.6$ $\mathrm{kg} / \mathrm{m}^{2}$ ). Os sujeitos realizaram 3 CVIM com exercícios de agachamento a $70^{\circ}$ e $90^{\circ}$ de flexão de joelho. $\mathrm{O}$ protocolo de coleta de dados ocorreu em dois dias não consecutivos: $1^{\circ}$ dia - mensurações antropométricas e realização de três CVIM a $70^{\circ} ; 2^{\circ}$ dia - realização de três CVIM a $90^{\circ}$. Para a normalização dos dados foi adotada a média dos valores em RMS. Os procedimentos metodológicos seguiram as recomendações da ISEK. Os resultados não mostraram diferenças significativas $(p>.05)$ entre as angulações para: VL $70^{\circ}$ vs VM $70^{\circ}$; VL $90^{\circ}$ vs VM 90; VL $70^{\circ}$ vs VL $90^{\circ}$; VM $70^{\circ}$ vs VM $90^{\circ}$. Estes resultados permitem concluir existir um equilíbrio na atividade elétrica entre o vasto lateral e o vasto medial durante a realização das CVIM em sujeitos destreinados.
\end{abstract}

Palavras-chave: EMG, agachamento, vasto medial, vasto lateral

ABSTRACT

The aim of the present study was to analyze the electric activity of the vastus medialis (VM) and vastus lateralis (VL) in two differing angles $\left(70^{\circ}\right.$ and $90^{\circ}$ of knee flexion) during maximum voluntary contraction in squat exercise. Fifteen male subjects composed the sample of the study. As criteria they were untrained for more than 12 months (age of $28.0 \pm 4.6$ years, height of $180.0 \pm 5.2 \mathrm{~cm}$, weight of $80.0 \pm 3.2 \mathrm{~kg}$ and body index mass of $24.2 \pm 1.6 \mathrm{~kg} / \mathrm{m}^{2}$ ). Subjects accomplished three maximum voluntary isometric contractions (MVIC) in squat exercise with $70^{\circ}$ and $90^{\circ}$ of knee flexion and separate at least in $72 \mathrm{~h}$ each. Data normalization occurred by average of values in RMS. Procedure adopted followed ISEK's recommendations. No significant differences $(p>.05)$ were found between the angles VL $70^{\circ}$ vs $\mathrm{VM} 70^{\circ}$, VL $90^{\circ}$ vs VM $90^{\circ}$, VL $70^{\circ}$ vs VL $90^{\circ}$, and VM $70^{\circ}$ vs VM $90^{\circ}$. Results allowed for the conclusion that there seems to exist a balance in electric activity between VL and VM during MVIC with $70^{\circ}$ and $90^{\circ}$ of knee flexion in untrained subjects.

Keywords: EMG, squat exercise, vastus lateralis, vastus medialis

Submetido: 19.10.2009 | Aceite: 01.08.2010

Alex Souto Maior. Departamento de Educação Física - Universidade Castelo Branco (UCB) - Rio de Janeiro, Brasil.

Leonardo Marmelo. Departamento de Educação Física - Universidade Plínio Leite (UNIPLI) - Niterói, RJ, Brasil. Silvio Marques-Neto. Departamento de Educação Física - Centro Universitário da Cidade (UniverCidade) - Rio de Janeiro, RJ, Brasil.

Endereço para correspondência: Alex Souto Maior, Av. Marechal Djalma Ribeiro, 25 - Apt 103, Recreio dos Bandeirantes, CEP: 22790-790 Rio de Janeiro - Brasil.

E-mail: alex.bioengenharia@terra.com.br 
O movimento de agachar é um padrão mecânico natural do desenvolvimento motor. Contudo, em relação ao treinamento físico, o exercício de agachamento, tem sido utilizado, com ou sem equipamentos específicos, para aplicação no desempenho físico e na reabilitação de membros inferiores em atletas, jovens e adultos (Escamilla, 2001).

O movimento isotônico de agachamento proporciona movimentos ascendentes e descendentes. Desta forma, a realização deste exercício sem uma prévia adaptação ao nível neural pode proporcionar mudanças agudas no comprimento das unidades músculo-tendão e alterar a habilidade de detectar (propriocepção aferente) e responder (atividade muscular eferente) às mudanças imediatas no ajuste postural (Behm, Bambury, Cahill, \& Power, 2004; Escamilla, 2001; Maior \& Alves, 2003). Os centros sensório-motores fornecem um relato preciso da situação do músculo para o nível espinhal. Desta forma, com a progressão do treinamento, os indivíduos treinados realizam movimentos que exijam grau significativo de coordenação intra e intermuscular e que envolvam interações automáticas do comando motor voluntário e postural dos grupamentos musculares superiores e inferiores (Alkner, Tesch, \& Berg, 2000; Bloem, Allum, Carpenter, \& Honegger, 2000; Maior \& Alves, 2003).

O grupo muscular do quadríceps é o mais volumoso e potente grupo muscular do corpo humano, consequentemente, constitui a maior parte da massa muscular da região anterior e medial da coxa (Escamilla, 2001; Ribeiro, Loss, Cañeiro, Lima, \& Martinez, 2005). Este grupo muscular apresenta quatro músculos em sua constituição: vasto lateral (VL), vasto medial (VM), vasto intermédio (VI) e reto da coxa (RC). O equilíbrio na atividade muscular entre o VL e o VM é visto como essencial para uma relação artrocinemática adequada entre a patela e a tróclea femoral (Maior, Moraes, Santos, \& Simão, 2006; Maior, Simão, Salles, Miranda, Costa, 2009; Ribeiro et al., 2005). Entretanto, dos quatro músculos do quadríceps, o RC, apresenta maior ação na articulação do quadril, enquanto, o VI é visualizado quando se rebate o músculo reto da coxa. Contudo, sugerem que a boa funcionalidade dinâmica dos músculos do quadríceps pode ser determinante na prevenção e/ou na limitação da severidade de lesões dos tecidos moles (Alkner et al., 2000; Isear, Jerome, Erickson, \& Worrell, 1997).

Medidas confiáveis para detecção de respostas neuromusculares são observadas com a utilização da electromiografia (EMG). A EMG tem a função de investigar a atividade elétrica do músculo por meio de sinal espectral (De Luca, 1997; Escamilla, 2001). Alguns fatores podem influenciar o sinal eletromiográfico, tais como: a espessura do tecido adiposo, distância entre os eletrodos, ativação das unidades motoras e impedância da pele (De Luca, 1997).

A associação entre exercícios de agachamento e EMG não mostraram diferenças significativas na atividade elétrica do VL e VM (Anderson, Courtney, \& Carmell, 1998). Contudo, foi verificada maior atividade electrica destes músculos com o aumento da flexão do joelho $\left(0-30^{\circ}, \quad 0-60^{\circ}, \quad 0-90^{\circ}\right)$ (Anderson et al., 1998). Outros estudos comentam que a atividade do quadríceps mantém-se constante perante as angulações 80-90 de flexão de joelho (Stuart, Meglan, Lutz, Growney, \& An, 1996). Consequentemente, quando a flexão de joelho é realizada abaixo de $90^{\circ}$ proporciona redução do potencial de recrutamento com menor ativação dos músculos do quadríceps (Escamilla, 2001). Dessa forma, o objetivo do estudo foi analisar e comparar a atividade contrátil dos músculos vasto lateral e vasto medial, por meio da EMG, em indivíduos destreinados submetidos ao exercício de agachamento bilateral.

\section{MÉTODO}

\section{Amostra}

A amostra do estudo foi composta de 15 sujeitos do sexo masculino voluntários, aparentemente saudáveis, com média de idades de $28.0 \pm 4.6$ anos, estatura de $180.0 \pm 5.2$ 
$\mathrm{cm}$, massa corporal de $80.0 \pm 3.2 \mathrm{~kg}$ e índice de massa corporal (IMC) de $24.2 \pm 1.6 \mathrm{~kg} / \mathrm{m}^{2}$. Os indivíduos selecionados eram destreinados há mais de 12 meses em relação ao treinamento de força.

Para melhor discriminar a amostra foram utilizados os seguintes critérios de exclusão para os indivíduos participantes do estudo: a) portadores de cardiopatia; b) portadores de lesões articulares nos últimos seis meses; c) portadores de contratura muscular nos últimos seis meses; d) submissão a cirurgias articulares nos últimos 12 meses; e) portadores de instabilidade acentuada nos joelhos e tornozelos; g) portadores de hérnia discal; e, h) portadores de formas severas de doenças articulares degenerativas.

\section{Instrumentos e Procedimentos}

Antes da coleta de dados, todos responderam aos itens do questionário Par-Q. Os indivíduos assinaram $O$ termo de consentimento para pesquisa com seres humanos, conforme Resolução no 196/96 do Conselho Nacional de Saúde do Brasil. O procedimento metodológico foi aprovado pelo Comitê de Ética para Pesquisa com Seres Humanos da Universidade Plínio Leite.

A medida da massa corporal foi realizada por uma balança digital (Filizola $\left.{ }^{\circledR}\right)$ e a estatura foi mensurada por um estadiômetro graduado em milímetros (Sanny®). O IMC foi baseado na equação da Organização Mundial de Saúde, em que a média de todos os indivíduos participantes do estudo mostrou-se dentro dos padrões de normalidade.

Os sujeitos do estudo realizaram execuções do movimento dois dias antes da coleta de dados para familiarização ao exercício de agachamento. As mensurações com o EMG foram realizadas em dois dias de treinamento não consecutivos (intervalo de $72 \mathrm{~h}$ entre os dias). No $1^{\circ}$ dia foram realizadas as medidas antropométricas e a mensuração da atividade elétrica do VL e VM a partir de três contrações voluntárias isométricas máximas (CVIM), de 10 segundos cada, no exercício de agachamento a $70^{\circ}$ de flexão de joelho. No $2^{\circ}$ dia, os sujeitos seguiram os padrões de medida do $1^{\circ}$ dia, porém realizando o movimento a $90^{\circ}$ de flexão de joelho. A realização das 3 CVIM foi utilizada para confiabilidade da medida. Para o procedimento de análise dos sinais biológicos foi utilizada a maior CVIM obtida. É importante referir que $\mathrm{o}$ aquecimento específico foi realizado previamente a cada sessão com duas séries de 15 repetições sem sobrecarga e o intervalo de recuperação entre cada CVIM foi de três minutos.

O exercício utilizado durante a coleta de dados foi o agachamento bilateral no Smith Machine. Para melhor discriminar a realização do exercício, estabeleceram-se as seguintes etapas de execução: posição inicial e fase concêntrica. A fase excêntrica foi realizada a partir do final da fase concêntrica. i) Posição inicial - $\mathrm{O}$ indivíduo em pé, pernas paralelas com um pequeno afastamento lateral entre 30 e $40 \mathrm{~cm}$, com os joelhos em extensão, com os cotovelos em flexão alinhados com os ombros, segurando a barra com a carga do treinamento apoiada na linha do trapézio; ii) Fase concêntrica - A partir da fase excêntrica $\left(70^{\circ}\right.$ ou $90^{\circ}$ de flexão do joelho dependendo da etapa da coleta de dados), realizou-se extensão completa dos joelhos e quadris. Para a realização do exercício de agachamento foi utilizado o equipamento Smith Machine (Life Fitness ${ }^{\circledR}$ - EUA).

Para a precisão da medida dos ângulos articulares determinados $\left(70^{\circ}\right.$ e $\left.90^{\circ}\right)$ foi utilizado um goniômetro digital (EMG System do Brasil ${ }^{\circledR}$, São José dos Campos - SP), em que o registo ocorreu através de um sensor fornecido por um sinal elétrico correspondente ao movimento angular.

Objetivando reduzir a margem de erro durante a realização dos testes, foram adotadas as seguintes estratégias: i) instruções padronizadas foram oferecidas antes do teste, de modo a que o avaliado estivesse ciente de toda a rotina que envolve a coleta de dados; ii) o avaliado foi instruído sobre a técnica de execução do exercício; iii) o avaliador estava 
atento quanto à posição adotada pelo praticante no momento da medida. Pequenas variações no posicionamento das articulações envolvidas na ação poderiam recrutar outros músculos, distanciando do foco específico da pesquisa, possibilitando interpretações erróneas dos escores obtidos; iv) foi estipulada a posição fixa de $0^{\circ}$ de abdução dos pés evitando, assim, a diferenciação na angulação do tornozelo do indivíduo nas tentativas durante o teste; e v) durante o teste os indivíduos mantiveram a base de suporte entre 30 e $40 \mathrm{~cm}$ (pés paralelos).

A EMG foi utilizada na mensuração da atividade elétrica dos músculos VL e VM em relação às distintas angulações $\left(70^{\circ}\right.$ e $\left.90^{\circ}\right)$ da flexão de joelho. Desta forma, para uma melhor detecção, amplificação e apresentação das alterações elétricas que ocorrem quando o músculo se contrai, os sinais da EMG foram amplificados $(1000 \times)$, filtrados (filtro de passa-banda entre $20-500 \mathrm{~Hz}$ ), digitalizados a uma taxa de 2000 pontos por segundo por um equipamento de eletromiografia de 4 canais da EMG Systems do Brasil® (São José dos Campos-SP, Brasil) e armazenados no computador para análises posteriores através da conversão analógica digital. Foi utilizada para análise a raiz quadrada da média quadrática da ativação elétrica (RMS) que corresponde à quantidade de sinal contínuo capaz de conter a mesma quantidade de energia (Stuart et al., 1996). Os sinais EMG obtidos em microvolts $(\mu \mathrm{V})$ foram digitalmente retificados e integrados durante $o$ período de $500 \mathrm{~ms}$ determinado a partir do início da CVIM. A normalização dos dados ocorreu a partir do valor de pico obtido na CVIM. O processamento dos sinais biológicos ocorreu com a exclusão do $1^{\circ}$ segundo e do último segundo, consequentemente, foi utilizada para análise uma janela de 8 segundos através do software Matlab (Matlab version 6.0, Mathworks, Massachusetts USA). Os gráficos do sinal RMS por tempo dos músculos vasto lateral e vasto medial foi realizado pelo software Graph Pad 5.0 (Massachusetts, USA).
Os grupos musculares analisados pela EMG foram: i) região central do vasto lateral (VL) aproximadamente a $2 \mathrm{~cm}$ do epicôndilo lateral do fêmur com a inclinação de $50.4^{\circ}$; ii) região do ventre muscular do vasto medial a uma inclinação de $55^{\circ}$ (VM) (Bevilaqua-Grossi, Felício, Simões, Coqueiro, \& Monteiro-Pedro, 2005). Para garantir a confiabilidade e precisão dos sinais eletromiográficos foi realizado o procedimento para minimização da impedância da pele, que incluiu retirada dos pêlos e limpeza da pele com álcool para melhor aplicação dos eletrodos.

Foram utilizados eletrodos ativos de diferenciais simples de superfície composta por duas barras paralelas de $\mathrm{Ag}-\mathrm{AgCl}$ retangulares, cada um com $1 \mathrm{~cm}$ de comprimento, $1 \mathrm{~mm}$ de largura e distância de $1 \mathrm{~cm}$ entre si, envolvidas em uma cápsula de poliuretano contendo um microcircuito elétrico. Os eletrodos foram posicionados no membro dominante (lado direito) do sujeito. Esses eletrodos foram acoplados a um pré-amplificador diferencial bipolar e fixados na pele (centro do ventre de cada músculo analisado) à distância de dois centímetros entre eles em todos os grupos musculares analisados. Assim, foram suficientes para envolverem todo o comprimento dos grupamentos mensurados. Para melhor interpretação das medidas espectrais com EMG, os eletrodos foram posicionados em paralelo com a fibra muscular do membro inferior dominante em que o eletrodo de aterramento ficou posicionado no punho contra-lateral ao membro inferior exposto aos eletrodos de ativos. Todo o procedimento adotado neste estudo respeitou as recomendações da International Society of Electrophysiology and Kinesiology (ISEK).

\section{Análise Estatística}

Os resultados das variáveis analisadas (análise por tempo - variação da escala de 0 a 10 segundos) foram apresentados como média e desvio padrão (descrição da amostra). Foi utilizado teste de Wilcoxon (não paramétrico) para verificação de diferenças significativas 
entre o comportamento dos dois músculos (vasto lateral e medial). Para a verificação do coeficiente de correlação foi utilizado o $r$ de Pearson. Em toda análise foi utilizado o software Graph Pad Prisma 5 e adotado um nível de significância de $5 \%(p<.05)$.

\section{RESULTADOS}

Os resultados não demonstraram diferenças significativas $(p>.05)$ entre as angulações: VL $70^{\circ}$ vs VM $70^{\circ}$; VL $90^{\circ}$ vs VM $90^{\circ}$; VL $70^{\circ}$ vs VL $90^{\circ}$; VM $70^{\circ}$ vs $\mathrm{VM} 90^{\circ}$ (ver figura $1 \mathrm{~A}$ e B). Mesmo não ocorrendo diferença intra e intergrupos, a atividade elétrica do vasto lateral apresentou aumento percentual de $8.3 \%$ em relação ao vasto medial no exercício de agachamento realizado no ângulo de $70^{\circ}$. Entretanto, no exercício de agachamento no ângulo de $90^{\circ}$ foi verificado um aumento percentual de $8.8 \%$ da atividade elétrica do vasto lateral quando comparado ao vasto medial. O coeficiente de correlação intraangulações revelou uma correlação significativa entre o vasto lateral e medial (ver figura 1C e D). Contudo, para as análises inter-angulações (ver figura 2A e B) no mesmo grupo muscular $\left(\mathrm{VL} 70^{\circ} \times \mathrm{VL} 90^{\circ}\right.$ e VM $\left.70^{\circ} \times \mathrm{VM} 90^{\circ}\right)$ foram obtidos índices de correlação elevados $(r=.86$ e $r=.75$, respectivamente), quando comparados aos valores verificados intra-angulações.

\section{DISCUSSÃO}

O presente estudo pretendeu verificar a atividade elétrica do vasto medial (VM) e do vasto lateral (VL) em duas angulações distintas durante o exercício de agachamento.

Os resultados não revelaram diferenças significativas intra e inter-grupos musculares analisados no presente estudo. Contudo, foi verificada uma tendência de maior potencial de recrutamento e moderado coeficiente de correlação do VL $\left(\mathrm{VL} 70^{\circ} \times \mathrm{VL} 90^{\circ}, r=.86, r^{2}\right.$ $=.74)$ em relação ao $\mathrm{VM}\left(\mathrm{VM} 70^{\circ} \times \mathrm{VM} 90^{\circ}, r\right.$ $\left.=.75, r^{2}=.56\right)$ entre ambas as angulações utilizadas no estudo. Nossos resultados corroboram com estudos previamente publicados, ou seja, nestes estudos foi verificada a atividade elétrica similar do VL e VM durante o exercício de agachamento (Bevilaqua-Grossi et al., 2005; Mirzabeigi,
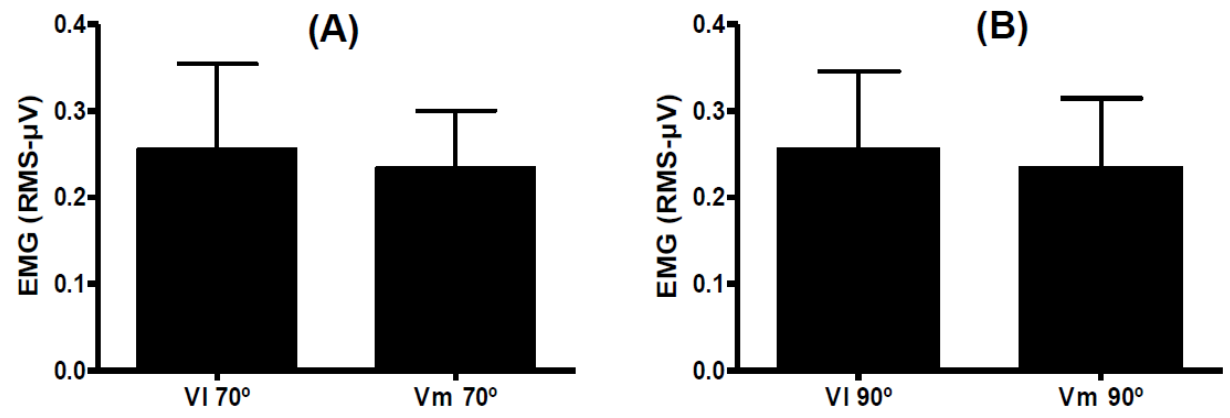

(C)

(D)
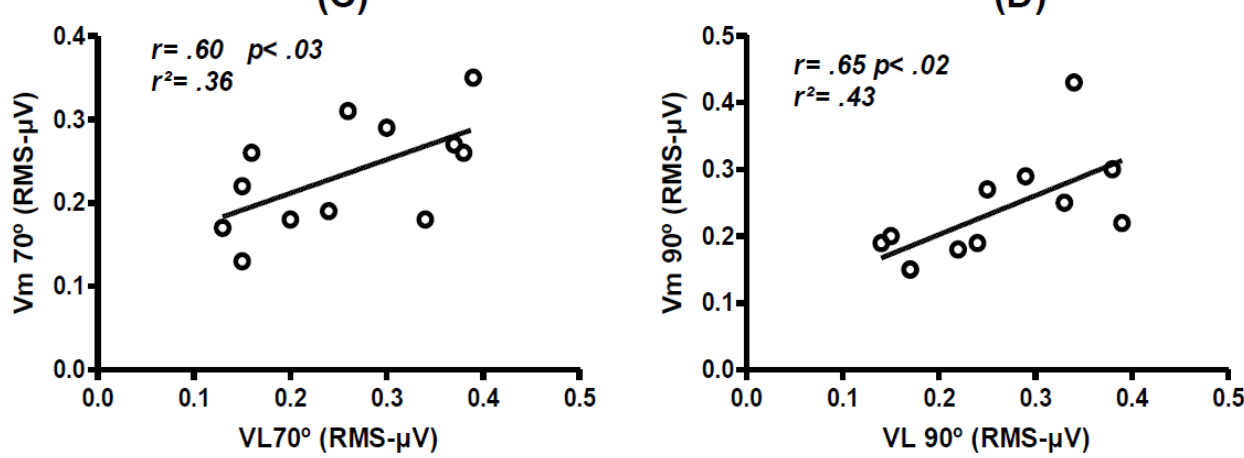

Figura 1. Dados expressos em média e desvio-padrão em relação às angulações e ao comportamento do VL e VM durante o exercício de agachamento 
(A)
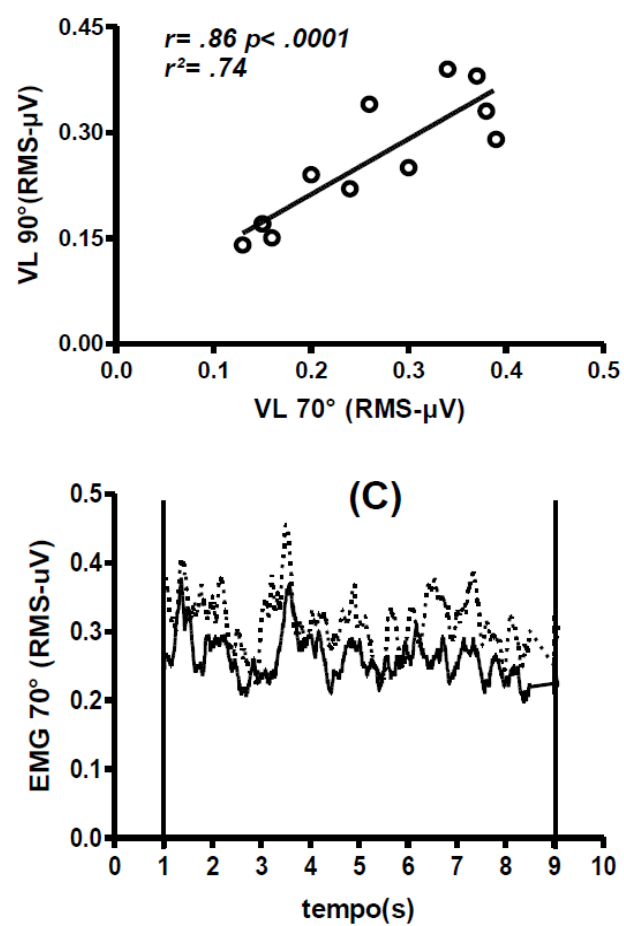

(B)

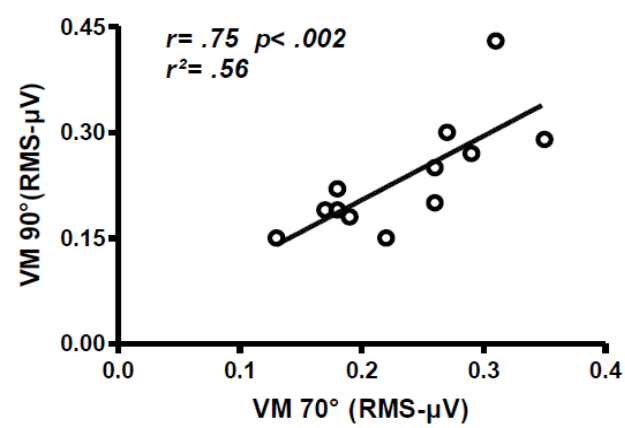

(D)

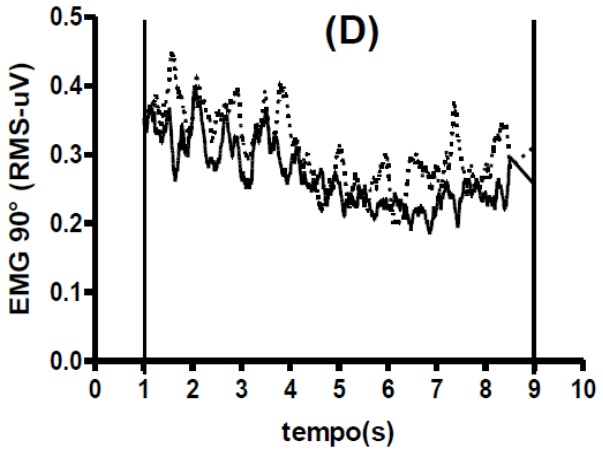

Figura 2. Comportamento do traçado do EMG com o exercício de agachamentoa $70^{\circ}$ (figura A) e $90^{\circ}$ (figura B). Nota: Linha tracejada - Vasto Lateral; Linha contínua - Vasto Medial

Jordan, Gronley, Rockowitz, \& Perry, 1999; Wilk, Escamilla, Fleisig, Barrentine, Andrews, \& Boyd, 1996).

No estudo de Isear et al. (1997) foi investigado o comportamento dos grupos musculares dos membros inferiores durante a contração voluntária máxima (CVM) em exercício de agachamento. Neste estudo o EMG mostrou alta atividade elétrica do VM e do VL, porém sem diferenças significativas inter-grupos musculares $(\mathrm{VM} \times \mathrm{VL})$. $\mathrm{O}$ estudo de McBride, Deane e Nimphius (2007) também apresenta conclusões que apontam nesse sentido. Eles submeteram oito sujeitos a realizarem exercícios de agachamento com e sem flexibilidade prévia e não verificaram diferenças na atividade elétrica dos músculos VM e VL em ambas as situações. Earl, Schmitz e Arnold (2001) também não verificaram diferença significativa entre o potencial de recrutamento dos músculos VM e VL, em atletas, durante o exercício de agachamento $0 \mathrm{a}$ $30^{\circ}$ de flexão de joelho.
A análise da EMG em outros exercícios que envolvem o quadríceps (cadeira extensora e leg press) foi verificada por Alkner et al. (2000) através de percentuais da CVM (20, 40, 60, 80 e $100 \%)$. Os resultados deste estudo mostraram que a actividade do VL manteve-se linear em todas as medidas da CVM, enquanto o VM apresentou aumento linear da atividade elétrica do músculo em cada fase da CVM. Complementarmente, outros estudos concluíram que o VM se mantém ativo ao longo de todo o arco do movimento durante a extensão do joelho (de 0 até $90^{\circ}$ na flexão do joelho) (Cintra \& Furlani, 1996). Entretanto, o músculo VL desempenha um papel recíproco e sincrônico na estabilização patelar (Wilk \& Reinold, 2001).

De acordo com os resultados do presente estudo e dos estudos anteriormente citados é importante comentar que o músculo VM restabelece a função normal da articulação femoropatelar e aumenta a contenção e estabilização dinâmica contra as forças que 
poderiam deslocar a patela lateralmente (Wilk \& Reinold, 2001). Outro fator associado é o posicionamento anatômico deste músculo, ou seja, o VM também apresenta uma pequena região de continuidade direta inserida no tendão patelar e não está somente inserido na borda medial da patela (Toumi et al., 2007). O papel do VL na estabilização da patela apresenta relevância pelo fato de estar localizado no septo lateral intermuscular unido à linha áspera do fêmur, consequentemente, fixado na borda supralateral da patela (Bevilaqua-Grossi, Monteiro-Pedro, Souza, Silva, \& Bérzin, 2004). Outro fator relevante em relação ao VL é a sua interação com o retinaculum lateral e o trato iliotibial (Bevilaqua-Grossi et al., 2004). Assim, a atividade elétrica similar destes dois grupos musculares (VL e VM) em distintas angulações durante a CVIM pode estar relacionada ao comportamento anatômico e biomecânico (mono-articulares) durante o movimento de agachar. Além disso, é importante comentar que existem semelhanças histoquímicas e morfológicas entre o VM e o VL (maior predominância de fibras musculares tipo II), principalmente entre o vasto medial oblíquo (VMO) e o vasto lateral longitudinal (VLL) (Travnik, Pernus, \& Erzen, 1995).

Parece que sujeitos destreinados associado à falta de adaptação do sistema nervoso ao exercício de agachamento revela aumento de recrutamento dos miopotenciais e maior disparo do potencial de ação com fator de ajuste postural (Escamilla, 2001; Johanson, 1988; Lee, 2004). Este procedimento ocorre pelo maior sincronismo das unidades motoras ativadas (maior excitabilidade dos motoneurônios) através do aumento da atividade dos motoneurônios alfa e gama (Aagaard, Simonsen, Andersen, Magnusson, \& DyhrePoulsen, 2002; Johanson, 1988; Lee, 2004). Assim, maior ação dos antagonistas, estabilizadores e sinergistas, com objetivo de buscar a homeostasia postural (Aagaard et al., 2002; Escamilla, 2001). Como fator de limitação do estudo seria importante também o controle da resposta dos músculos antagonistas do quadríceps e estabilizadores de tronco.

\section{CONCLUSÕES}

O presente estudo revelou que as CVIM nos exercícios de agachamento realizados em $70^{\circ} \mathrm{e}$ $90^{\circ}$ de flexão de joelho não apresentam diferenças na atividade elétrica dos grupos musculares do vasto lateral e medial. Entretanto, é importante ressaltar o incentivo a novas pesquisas relacionando novas angulações de flexão de joelho em diferentes exercícios que envolvam os músculos estabilizadores da patela.

\section{REFERÊNCIAS}

Aagaard, P., Simonsen, E. B., Andersen, J. L., Magnusson, P., \& Dyhre-Poulsen, P. (2002). Neural adaptation to resistance training: Changes in evoked V-wave and H-reflex responses. Journal of Applied Physiology, 92, 2309-2318.

Alkner, B. A., Tesch, P. A., \& Berg, H. E. (2000). Quadriceps EMG/force relationship in knee extension and leg press. Medicine \& Science in Sports \& Exercise, 32, 459-463.

Anderson, R., Courtney, C., \& Carmell, E. (1998). EMG analysis of the vastus medialis/vastus lateralis muscles utilizing the unloaded narrowand wide-stance squats. Journal of Sport Rehabilitation, 7, 236-247.

Behm, D. G., Bambury, A., Cahill, F., \& Power, K. (2004). Effect of acute static stretching on force, balance, reaction time, and movement time. Medicine \& Science in Sports \& Exercise, 36, 1397-1402.

Bevilaqua-Grossi, D., Felício, L. R., Simões, R., Coqueiro, K. R. R., \& Monteiro-Pedro, V. (2005). Avaliação eletromiográfica dos músculos estabilizadores da patela durante exercício isométrico de agachamento em indivíduos com síndrome da dor femoropatelar. Revista Brasileira de Medicina do Esporte, 11, 159-163.

Bevilaqua-Grossi, D., Monteiro-Pedro, V., Souza, G. C., Silva, Z., \& Bérzin, F. (2004). Contribution to the anatomical study of the oblique portion of the vastus lateralis muscle. Brazilian Journal of Morphological Sciences, 21 (1), 47-52.

Bloem, B. R., Allum, J. H. J., Carpenter, M. G., \& Honegger, F. (2000). Is lower leg propriocep- 
tion essential for triggering human automatic postural response? Experimental Brain Research, 130, 375-391.

Cintra, A. I. D., \& Furlani, J. (1996). Electromyography study of quadriceps femoris in man. Electromyography and Clinical Neurophysiology, 21, 539-554.

De Luca, C. J. (1997). Use of the surface electromyography in biomechanics. Journal of Applied Biomechanics, 13, 135-163.

Earl, J. E., Schmitz, R. J., \& Arnold, B. L. (2001). Activation of VMO and VL during dynamic mini-squat exercises with and without isometric hip adduction. Journal of Electromyography and Kinesiology, 11, 381-386.

Escamilla, R. F. (2001). Knee biomechanics of the dynamic squat exercise. Medicine $\mathcal{E}$ Science in Sports \& Exercise, 33 (1), 127-141.

Isear, R., Jerome, A., Erickson, C., \& Worrell, T. W. (1997). EMG analysis of lower extremity muscle recruitment patterns during an unloaded squat. Medicine \& Science in Sports \& Exercise, 29, 532-539.

Johanson, H. (1988). Rubrospinal and rubrobulbospinal influences on dynamic and static gama-motoneurons. Behavioural Brain Research, 28, 97.

Lee, T. H. (2004). Lifting strengths decrease with the extent of squat postures. Journal of Back and Musculoskeletal Rehabilitation, 17(3/4), 105-109.

Maior, A. S., \& Alves, A. (2003). A contribuição dos fatores neurais em fases iniciais do treinamento de força: Uma revisão bibliográfica. Motriz, 9(3), 161-168.

Maior, A. S., Moraes, E. R., Santos, T. M., \& Simão, R. (2006). Análise da força muscular em indivíduos treinados na plataforma de instabilidade. Revista Brasileira de Ciência $e$ Movimento, 14(2), 41-48.

Maior, A. S., Simão, R., Salles, B. F., Miranda, H., \& Costa, P. B. (2009). Neuromuscular activity during the squat exercise on an unstable platform. Brazilian Journal of Biomotricity, 3(2), 121-129.

McBride, J. M., Deane, R., \& Nimphius, S. (2007). Effect of stretching on agonist-antagonist muscle activity and muscle force output during single and multiple joint isometric contractions. Scandinavian Journal of Medicine and Science in Sports, 17(1), 54-60.

Mirzabeigi, E., Jordan, C., Gronley, J. K., Rockowitz, N. L., \& Perry, J. Y. (1999). Isolation of the vastus medialis oblique muscle during exercise. The American Journal of Sports Medicine, 27, 50-53.

Ribeiro, D. C., Loss, J. F., Cañeiro, J. P. T., Lima, C. S., \& Martinez, F. G. (2005). Electromyographical analysis of the quadriceps during knee extension at different speeds. Acta Ortopédica Brasileira, 13,189-193.

Stuart, M. J., Meglan, D. A., Lutz, G. E., Growney, E. S., \& An, K. N. (1996). Comparison of intersegmental tibiofemoral joint forces and muscle activity during various closed kinetic chain exercises. The American Journal of Sports Medicine, 24, 792-799.

Toumi, H., Poumarat, G., Benjamin, M., Best, T., F'Guyer, S., \& Fairclough, J. (2007). New insights into the function of the vastus medialis with clinical implications. Medicine \& Science in Sports \& Exercise, 39(7), 1153-1159.

Travnik, L., Pernus, F., \& Erzen, I. (1995). Histochemical and morphometric characteristics of the normal human vastus medialis longus and vastus medialis obliquus muscles. Journal of Anatomy, 187(2), 403-411.

Wilk, K. E., \& Reinold, M. M. (2001). Principles of patellofemoral rehabilitation. Sports Medicine \& Arthritis Review, 9, 325-336.

Wilk, K. E., Escamilla, R. F., Fleisig, G. S., Barrentine, S. W., Andrews, J. R., \& Boyd, M. L. (1996). A comparison of tibiofemoral joint forces and electromyographic activity during open and closed kinetic chain exercises. The American Journal of Sports Medicine, 24, 518-527.

(cc) EY-No quando especificado em contrário e nos conteúdos retirados de outras fontes bibliográficas. 